KCL-PH-TH/2014-13, LCTS/2014-13, CERN-PH-TH/2014-060

\title{
Closing in on the Tip of the CMSSM Stau Coannihilation Strip
}

\author{
Nishita Desai ${ }^{1}$, John Ellis ${ }^{2,3}$, Feng Luo ${ }^{2}$ and Jad Marrouche ${ }^{4}$ \\ ${ }^{1}$ Institut für theoretische Physik, Universität Heidelberg, \\ Heidelberg 69120, Germany \\ ${ }^{2}$ Theoretical Particle Physics and Cosmology Group, Department of Physics, \\ King's College London, London WC2R 2LS, United Kingdom \\ ${ }^{3}$ Theory Division, CERN, CH-1211 Geneva 23, Switzerland \\ ${ }^{4}$ Physics Department, CERN, CH-1211 Geneva 23, Switzerland
}

\begin{abstract}
Near the tip of the $\tilde{\tau}$ coannihilation strip in the CMSSM with a neutralino LSP $\chi$, the astrophysical cold dark matter density constraint forces the $\tilde{\tau}-\chi$ mass difference to be small. If this mass difference is smaller than $m_{\tau}$, the $\tilde{\tau}$ may decay either in the outer part of an LHC detector - the 'disappearing track' signature - or be sufficiently long-lived to leave the detector before decaying - the long-lived massive charged-particle signature. We combine searches for these signatures with conventional $E_{T}^{\text {miss }}$ searches during LHC Run 1, identifying the small remaining parts of the CMSSM $\tilde{\tau}$ coannihilation strip region that have not yet been excluded, and discussing how they may be explored during Run 2 of the LHC.
\end{abstract}




\section{Introduction}

A major theme of the searches for new physics during Run 1 of the $\mathrm{LHC}$ at 7 and $8 \mathrm{TeV}$ in the centre of mass has been the search for supersymmetry via various experimental signatures [1,2]. The constraints imposed by the absences of any statistically-significant excesses of events with such signatures are frequently interpreted assuming that $R$ parity is conserved, in which case the lightest supersymmetric particle (LSP) could be present today as a cosmological relic [3] from the Big Bang, and many astrophysical constraints also come into play. Foremost among these is the density of cold dark matter [4,5], which imposes restrictions on supersymmetric model parameters that are often respected only in narrow strips in the supersymmetric parameter space. This is the case, for example, if it is assumed that the LSP is the lightest neutralino $\chi$. In particular, in the minimal supersymmetric extension of the Standard Model with soft supersymmetry-breaking parameters $m_{1 / 2}, m_{0}$ and $A_{0}$ assumed to be universal at the grand unification scale (the CMSSM) [6,7], the cold dark matter density constraint is respected along strips where $\chi$ coannihilation with the lighter $\tilde{\tau}$ slepton [8] or the lighter $\tilde{t}$ squark [9] is important, or where $\chi-\chi$ annihilation is enhanced by a directchannel Higgs resonance [6,10], or an enhanced Higgsino component in the composition of the LSP $\chi 11$.

As discussed in previous papers [12 14], the $\tilde{\tau}-\chi$ coannihilation strip region in the CMSSM is particularly accessible to supersymmetry searches at the LHC. However, complete exploration of this region requires a combination of different search strategies. As the tip of the $\tilde{\tau}-\chi$ coannihilation strip is approached at large $m_{1 / 2}$, the $\tilde{\tau}-\chi$ mass difference $\Delta m$ becomes very small. If $\Delta m>m_{\tau}$, the $\tilde{\tau}$ and other heavier sparticles decay rapidly into the LSP $\chi$, providing a classical $E_{T}^{m i s s}$ signature. However, if $\Delta m<m_{\tau}$ the $\tilde{\tau}$ lifetime becomes so long that it may decay in the outer part of a generic LHC detector - the 'disappearing track' signature - or even outside the detector altogether, in which case the $\tilde{\tau}$ would appear as a slow-moving massive, penetrating charged particle. Full exploration of the CMSSM $\tilde{\tau}-\chi$ coannihilation strip therefore requires a careful combination of searches for these signatures as well as for $E_{T}^{m i s s}$.

In a previous paper [12], the principal decay rates and the lifetime of the $\tilde{\tau}$ in the CMSSM when $\Delta m<m_{\tau}$ were re-evaluated, and the impact on the CMSSM of the Run 1 LHC searches for massive metastable charged particles were analyzed. Subsequently, updated LHC results from searches for such particles have been made available [15], as well as searches for $E_{T}^{\text {miss }}$ events [16 and disappearing tracks [19]. The purpose of this paper is to make a combined analysis of these different searches within the CMSSM, identify the remaining regions of the 
CMSSM $\tilde{\tau}$ coannihilation strip, and discuss how they may be explored in Run 2 of the LHC.

In Section 2 of this paper we first review relevant features of the $\tilde{\tau}$ coannihilation strip region within the CMSSM, which extends up to $m_{1 / 2} \sim 1300 \mathrm{GeV}$ for $\tan \beta=40$ and $A_{0}>0$. We then review the calculations of $\tilde{\tau}$ decays when $\Delta m<m_{\tau}$, which indicate that the dominant $\tilde{\tau}$ signature would be a massive metastable charged particle if $\Delta m \lesssim 1.2 \mathrm{GeV}$ and a disappearing track if $\Delta m \gtrsim 1.2 \mathrm{GeV}$.

In Section 3 we discuss the impacts of the relevant LHC Run 1 searches for new physics, including regions where the relic LSP density is less than the total cold dark matter density, as would be allowed if there is another component of the astrophysical cold dark matter. We first discuss the $E_{T}^{\text {miss }}$ searches, which exclude the relevant portions of the CMSSM parameter space where $\Delta m>m_{\tau}$ and $m_{1 / 2}<780 \mathrm{GeV}$. For $\tan \beta=10$, these searches exclude the portion of the $\tilde{\tau}$ coannihilation strip where $\Delta m \gtrsim 3 \mathrm{GeV}$, whereas $\Delta m$ as large as $9 \mathrm{GeV}$ can be allowed for $\tan \beta=40$. We then update our previous analysis of the metastable $\tilde{\tau}$ case, finding that the most recent LHC Run 1 search for such particles excludes $m_{1 / 2} \lesssim 800 \mathrm{GeV}$ to $\lesssim 1100 \mathrm{GeV}$ for $\Delta m \lesssim 1.2 \mathrm{GeV}$, depending on the value of $\tan \beta$ and $A_{0}$. We then analyze the impact of the disappearing track search on the intermediate band where $m_{\tau}>\Delta m \gtrsim 1.2 \mathrm{GeV}$, using Pyтнia 8 20,21] to simulate $\tilde{\tau}$ decays in an LHC detector outside the beam-pipe. We find that this search is weaker than the other constraints, yielding $m_{1 / 2} \gtrsim 400 \mathrm{GeV}$.

In Section 4 we discuss the interplay of these different searches, as well as the constraints from the observed value of the Higgs mass $m_{h}$ [22,23], calculated using the recently-released FeynHiggs 2.10.0 24.

In Section 5 we consider the sensitivities of LHC Run 2 searches with 300/fb of integrated luminosity at $14 \mathrm{TeV}$ in the centre of mass. The conventional $E_{T}^{\text {miss }}$ searches should have sufficient sensitivity to find evidence for supersymmetry or to exclude the coannihilation region of the CMSSM if $\Delta m>m_{\tau}$. Likewise, searches for massive metastable charged particles should be able to find evidence for the $\tilde{\tau}$ or to exclude the coannihilation region of the CMSSM if $\Delta m \lesssim 1.2 \mathrm{GeV}$. However, simple extrapolation of the current disappearing track searches indicates that they would have insufficient sensitivity to exclude or find evidence for supersymmetry if $m_{\tau}>\Delta m \gtrsim 1.2 \mathrm{GeV}$, so we consider ways in which the sensitivity of future such searches could be enhanced.

Finally, Section 6 summarizes our conclusions. 


\section{The $\tilde{\tau}$ Coannihilation Strip and its Decays within the CMSSM}

\subsection{Anatomy of the Stau Coannihilation Strip Region}

The focus of our attention in this paper is the CMSSM, in which $R$ parity is conserved and it is assumed that universal soft supersymmetry-breaking parameters $m_{1 / 2}, m_{0}$ and $A_{0}$ are input at the GUT scale. We assume that the stable LSP is the lightest neutralino $\chi$, giving priority to the CMSSM parameter region near the strip where its astrophysical relic density is brought into the range $0.115<\Omega_{\chi} h^{2}<0.125[5]$ that is acceptable within conventional cosmology by coannihilation with the lighter tau-slepton $\tilde{\tau}$ and other, heavier sleptons, but also considering smaller values of $\Delta m$ that yield lower values of $\Omega_{\chi} h^{2}$. Our objective is to study the extent to which this simplest supersymmetric scenario has been explored with data from Run 1 of the LHC at 7 and $8 \mathrm{TeV}$ in the centre of mass, and the extent to which it can be explored further with future LHC data at $14 \mathrm{TeV}$. As we discuss, even this simplest scenario has rich phenomenological possibilities beyond the standard $E_{T}^{m i s s}$ signatures, posing challenges for its complete exploration.

As is well-known, as $m_{1 / 2}$ increases toward the tip of the stau coannihilation strip the $\tilde{\tau}-\chi$ mass difference $\Delta m$ decreases monotonically towards zero, which is attained at $m_{1 / 2}=$ $\mathcal{O}(1000) \mathrm{GeV}$, the maximum value of $m_{1 / 2}$ depending on the values of $\tan \beta$ and $A_{0}$. In this paper, we use consistently SoftSUSY 3.3.7 25 to calculate the sparticle spectrum, and the latter is passed to MicrOMEGAs 3.5.5 27 to calculate $\Omega_{\chi} h^{2}$. Fig. 1 displays bands with $\Delta m \leq 5 \mathrm{GeV}$ for values of $m_{1 / 2}$ close to the tips of the coannihilation strips for $\tan \beta=10$ (upper panels) and 40 (lower panels), in each case for the two choices $A_{0}=0$ (left panels) and $2.5 m_{0}$ (right panels). The choices of $\tan \beta$ are representative of the larger and smaller values found in the coannihilation region in a recent global analysis of the CMSSM parameter space [28], and the restriction to $A_{0} \geq 0$ is motivated by the Higgs boson mass $m_{h}$ measured at the LHC, which is easier to reproduce for positive values of $A_{0} 1$. The coannihilation strips where $0.115<\Omega_{\chi} h^{2}<0.125$ are shown as pink bands. We see that the strips for $\tan \beta=10$ terminate when $\Delta m \rightarrow 0$ at $m_{1 / 2} \simeq 900$ to $950 \mathrm{GeV}$, with little sensitivity to $A_{0}$, whereas the strips for $\tan \beta=40$ and $A_{0}=0\left(2.5 m_{0}\right)$ extend to larger $m_{1 / 2} \simeq 1150$ to $1200 \mathrm{GeV}$ $(1300$ to $1350 \mathrm{GeV})$. We also see that $\Delta m$ drops below $m_{\tau}$ for $m_{1 / 2} \simeq 800$ to $850 \mathrm{GeV}$ for $\tan \beta=10$, and $m_{1 / 2} \simeq 1050$ to $1100 \mathrm{GeV}(1200$ to $1250 \mathrm{GeV})$ for $\tan \beta=40$, respectively.

\footnotetext{
${ }^{1}$ These choices of $\tan \beta$ and $A_{0}$ are also used in 12. However, to avoid confusion when the reader compares the results of this paper with the previous one 12, where the SSARD code 26 was used, please note that here we use the opposite convention for the sign of $A_{0}$.
} 

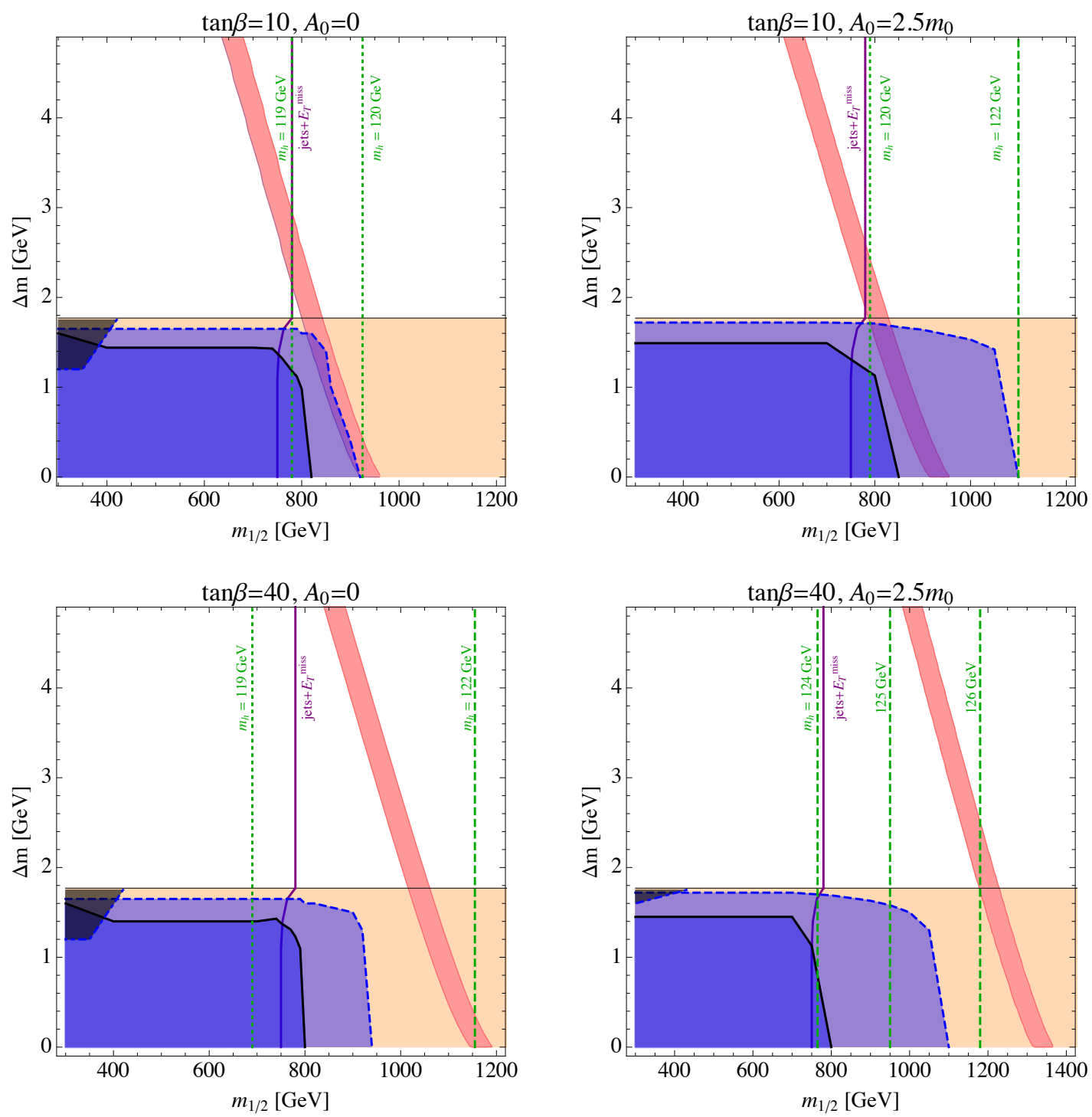

Figure 1: Overviews of the regions of the CMSSM parameter space with small mass difference $\Delta m \equiv m_{\tilde{\tau}}-m_{\chi}$ for $\tan \beta=10$ (upper panels) and $\tan \beta=40$ (lower panels), and for $A_{0}=0$ (left panels) and $2.5 m_{0}$ (right panels). The bands with $\Delta m<m_{\tau}$ are shaded beige, and the coannihilation strips where $0.125>\Omega_{\chi} h^{2}>0.115$ as calculated using SoftSUSY 3.3 .7 [25] coupled to MicrOMEGAs 3.5.5 [27] are shaded pink. The lower limit on $m_{1 / 2}$ from the ATLAS $E_{T}^{\text {miss }}$ search during Run 1 at the LHC [16] is represented in each panel by a maroon line, and contours of $m_{h}$ calculated using FeynHiggs 2.10.0 [24] are shown as green (dashed or dotted) lines. Parameter regions excluded by searches for the direct and total production of metastable charged particles [15] are shaded darker and lighter blue, respectively, and regions excluded by searches for particles leaving disappearing tracks are shaded grey (see Section 3 for details). 
The strips within which the relic LSP density $\Omega_{\chi} h^{2}$ falls inside the range allowed by the available astrophysical and cosmological data for the total cold dark matter density $\Omega_{C D M} h^{2}$ are quite narrow, since $\Omega_{C D M} h^{2}$ is tightly constrained, at the \% level, and the theoretical uncertainties in calculating $\Omega_{\chi} h^{2}$ are small within conventional Big Bang cosmology. However, we note that $\Omega_{\chi} h^{2}$ could be substantially smaller if the LSP is not the only important component of the cold dark matter. We therefore consider also the regions of the CMSSM parameter space with lower $\Omega_{\chi} h^{2}$ that have $\Delta m$ smaller than along the coannihilation strips displayed in Fig. 1 .

We return later to the other features exhibited in Fig. 1.

\subsection{Review of $\tilde{\tau}$ Decays}

The starting point for the calculation of $\tilde{\tau}$ decays in the CMSSM is the $\tilde{\tau}-\chi-\tau$ Lagrangian, which was derived in detail in the Appendix of [12]. If $\Delta m>m_{\tau}$, the dominant decay mode is $\tilde{\tau}^{-} \rightarrow \tau^{-} \chi$, which gives a $\tilde{\tau}$ lifetime many orders of magnitude smaller than 1 nanosecond. If $\Delta m<m_{\tau}$, the three- and four-body decay modes, $\tilde{\tau}^{-} \rightarrow a_{1}^{-}(1260) \nu_{\tau} \chi, \tilde{\tau}^{-} \rightarrow \rho^{-}(770) \nu_{\tau} \chi$, $\tilde{\tau}^{-} \rightarrow \pi^{-} \nu_{\tau} \chi, \tilde{\tau}^{-} \rightarrow \mu^{-} \bar{\nu}_{\mu} \nu_{\tau} \chi$ and $\tilde{\tau}^{-} \rightarrow e^{-} \bar{\nu}_{e} \nu_{\tau} \chi$, are the relevant ones, and their branching ratios varying with $\Delta m$, in particular. These channels close in sequence toward the tip of the coannihilation strip as $\Delta m \rightarrow 0$. Analytic expressions of the $\tilde{\tau}$ decay rates for these channels can be found in the Appendix of $[12$.

For $1.2 \mathrm{GeV} \lesssim \Delta m<m_{\tau}$, the $\tilde{\tau}$ lifetime is between order one and several hundred nanoseconds, so that the $\tilde{\tau}$ may decay in the outer part of the ATLAS and CMS detectors, and a disappearing track is the dominant $\tilde{\tau}$ signature. Over most of this range of $\Delta m$, $\tilde{\tau}^{-} \rightarrow \rho^{-}(770) \nu_{\tau} \chi$ is the dominant decay mode with a branching ratio varying between $\sim 29 \%$ and $\sim 37 \%$. The $\tilde{\tau}^{-} \rightarrow \pi^{-} \nu_{\tau} \chi$ branching ratio increases roughly linearly from $\sim 13 \%$ to $\sim 36 \%$ with the decrease of $\Delta m$, and it becomes the dominant mode at the lower end of $\Delta m$. The branching ratio of $\tilde{\tau}^{-} \rightarrow e^{-} \bar{\nu}_{e} \nu_{\tau} \chi$ is $\sim 18 \%$ to $20 \%$ over this $\Delta m$ range, and $\tilde{\tau}^{-} \rightarrow \mu^{-} \bar{\nu}_{\mu} \nu_{\tau} \chi$ is $\sim 1 \%$ smaller than the former. The $\tilde{\tau}^{-} \rightarrow a_{1}^{-}(1260) \nu_{\tau} \chi$ branching ratio is about the same size as each of the four-body decay modes at $\Delta m \sim m_{\tau}$, decreasing to $\sim 5 \%$ at $\Delta m \sim 1.5 \mathrm{GeV}$, and continually decreasing until its phase space vanishes at $\Delta m=m_{a_{1}}$. For $\Delta m \lesssim 1.2 \mathrm{GeV}$, the $\tilde{\tau}$ is sufficiently long-lived so that it leaves the detectors before decaying, and the signature would be a massive metastable charged particle.

The $\tilde{\tau}$ lifetime and decay branching ratios were plotted in Fig. 7 and 8 in [12. In those figures the $\chi$ was assumed to be pure bino-like with a mass of $300 \mathrm{GeV}$, and the $\tilde{\tau}_{L}-\tilde{\tau}_{R}$

mixing angle, $\theta_{\tilde{\tau}}$, was taken to be $\pi / 3$. These parameters were chosen in order to compare 
with the results of an earlier paper [29] where some differences in the $\tilde{\tau}$ decay calculations were found. We note that along $\tilde{\tau}$ coannihilation strip within the CMSSM, the $\chi$ is almost a bino, and the $\tilde{\tau}$ is almost right-handed. To see the effects of $\theta_{\tilde{\tau}}$ on the $\tilde{\tau}$ lifetime and decay branching ratios, we plot in the left panel of Fig. 2 the $\tilde{\tau}$ lifetime as a function of $\theta_{\tilde{\tau}}$ for the same $300 \mathrm{GeV}$ pure bino-like $\chi$ and for $\Delta m=1.2,1.3, \cdots, 1.7 \mathrm{GeV}$, and in the right panel we show the branching ratios $v s$. $\theta_{\tilde{\tau}}$ curves for the same $\chi$ parameters and for $\Delta m=1.5$ $\mathrm{GeV}$. In these plots, $\theta_{\tilde{\tau}}=\pi / 2$ corresponds to a pure right-handed $\tilde{\tau}$. We only show the plots for $\theta_{\tilde{\tau}} \in[0, \pi]$ because adding a $\pi$ to $\theta_{\tilde{\tau}}$ is equivalent to change the overall sign of the $\tilde{\tau}-\chi-\tau$ Lagrangian, and it has no effect for the $\tilde{\tau}$ decay rates calculations performed in [12. We can see that the $\tilde{\tau}$ lifetime strongly depends on $\theta_{\tilde{\tau}}$, but this dependence is not as strong as that of on $\Delta m$. On the other hand, the branching ratios only mildly depend on $\theta_{\tilde{\tau}}$, and this is also true for other choices of $\Delta m$ which we do not show here. Finally, we note that for a given $\Delta m$, the $\tilde{\tau}$ lifetime is roughly proportional to $m_{\chi}$ (so that it is roughly proportional to $m_{1 / 2}$ in the CMSSM due to the relation $m_{\chi} \sim 0.42 m_{1 / 2}$ ), while the branching ratios are not sensitive to $m_{\chi}$.
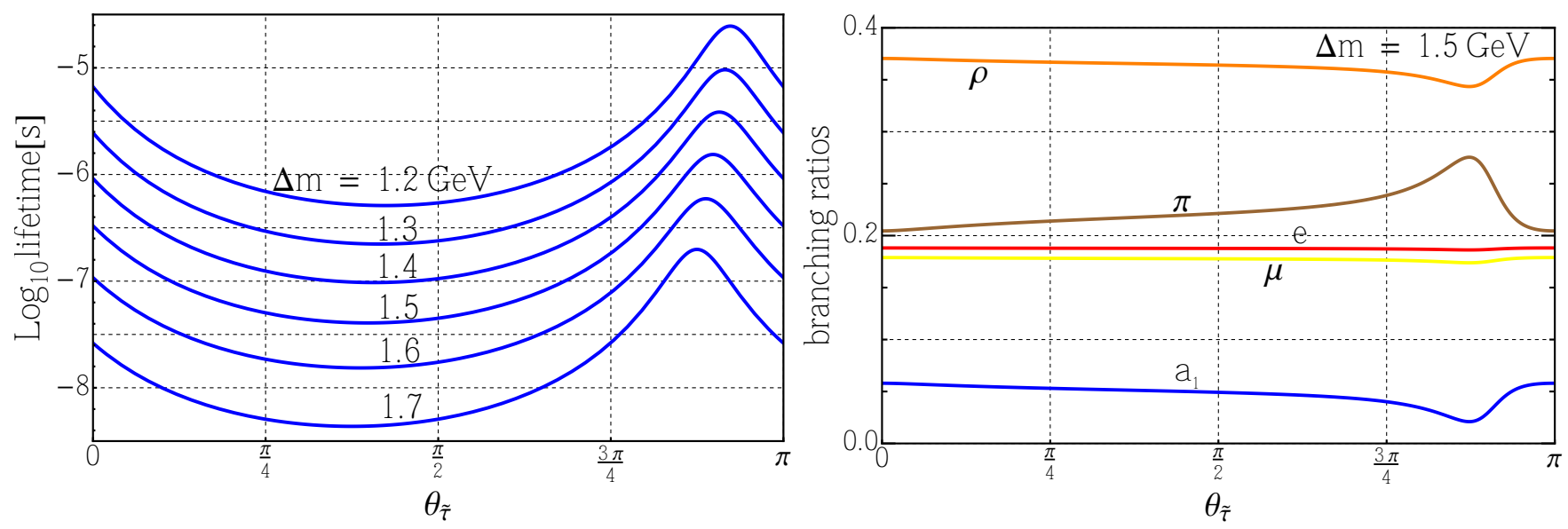

Figure 2: Left panel: the $\tilde{\tau}$ lifetime as a function of the $\tilde{\tau}_{L}-\tilde{\tau}_{R}$ mixing angle, $\theta_{\tilde{\tau}}$, for $\Delta m=$ $1.2,1.3, \cdots, 1.7 \mathrm{GeV}$. Right panel: the $\tilde{\tau}$ decay branching ratios as functions of $\theta_{\tilde{\tau}}$ for $\Delta m=$ $1.5 \mathrm{GeV}$. The blue, orange, brown, yellow, and red lines are for the final states with $a_{1}(1260)$, $\rho(770), \pi, \mu$, and e, respectively, indicated by the labels adjacent to the corresponding curves. In both panels, a pure bino-like $\chi$ with a mass of $300 \mathrm{GeV}$ is assumed.

Fig. 3 shows the $\tilde{\tau}$ lifetime contours as functions of $m_{1 / 2}$ and $\Delta m$, for the range $m_{1 / 2} \in$ $(300,1400) \mathrm{GeV}$ and $\Delta m \in(1.2,1.7) \mathrm{GeV}$. For all the four choices of the CMSSM parameters used in Fig. 1, within this small $\Delta m$ range the $\chi$ is almost a bino, and the $\tilde{\tau}$ is almost right- 
handed. Therefore, the $\tilde{\tau}$ lifetime essentially only depends on $\Delta m$ and $m_{\chi}$ (or, equivalently, $\left.m_{1 / 2}\right)$, so that the contours are almost identical for all the four choices of the CMSSM parameters. The widths of the contours in Fig. 3 span the dependence of the $\tilde{\tau}$ lifetime on $\tan \beta$ and $A_{0}$. One can check that the $\tilde{\tau}$ lifetimes at $m_{1 / 2} \sim 720 \mathrm{GeV}$ are consistent with the values shown in the left panel of Fig. 2 for $\theta_{\tilde{\tau}} \sim \pi / 2$.

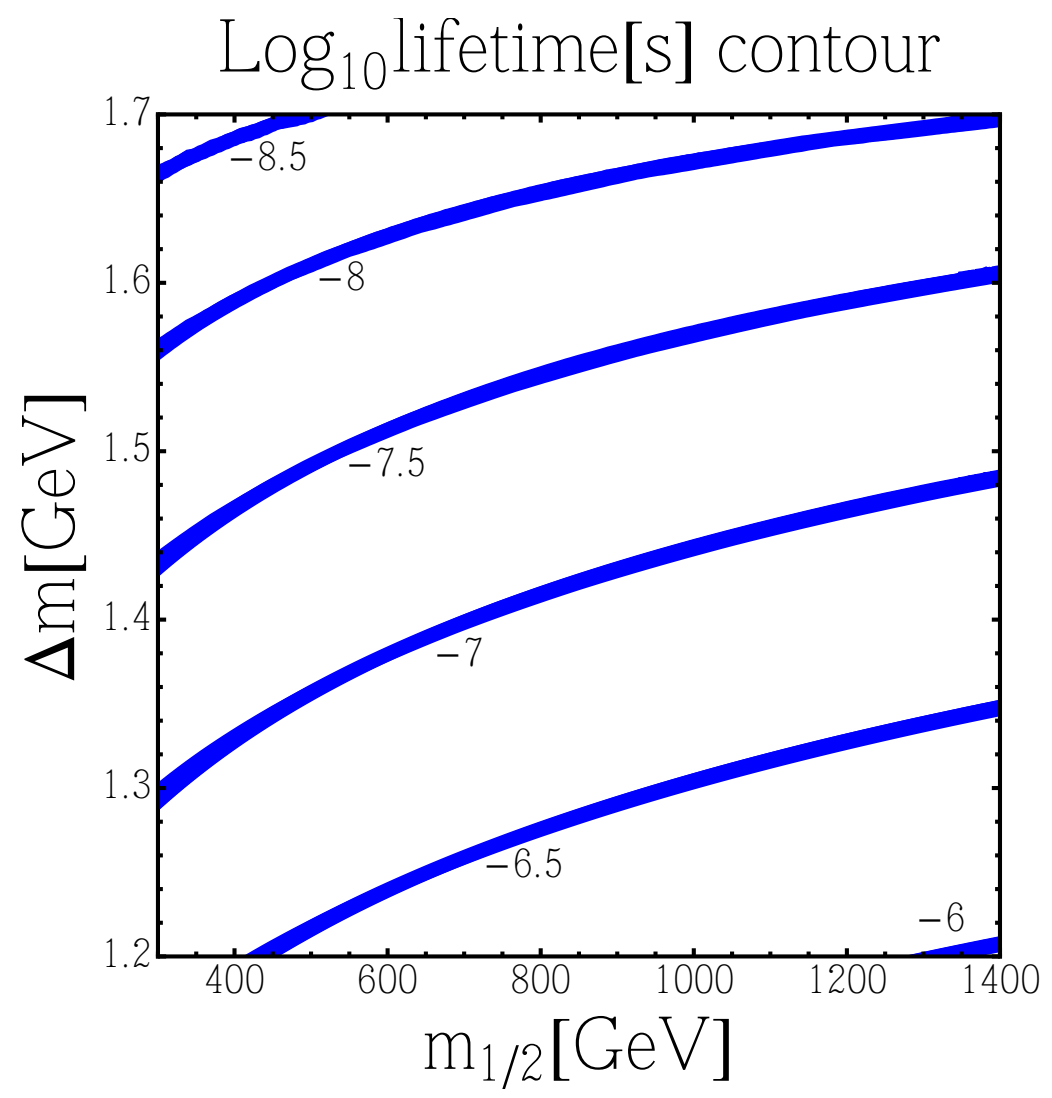

Figure 3: Some $\tilde{\tau}$ lifetime contours as functions of $m_{1 / 2}$ and $\Delta m$, for the four choices of the CMSSM parameters used in Fig. 1, namely, $\tan \beta=10,40$ and $A_{0}=0,2.5 m_{0}$. The widths of the contours span ranges of the $\tilde{\tau}$ lifetime for the different choices of $\tan \beta$ and $A_{0}$. 

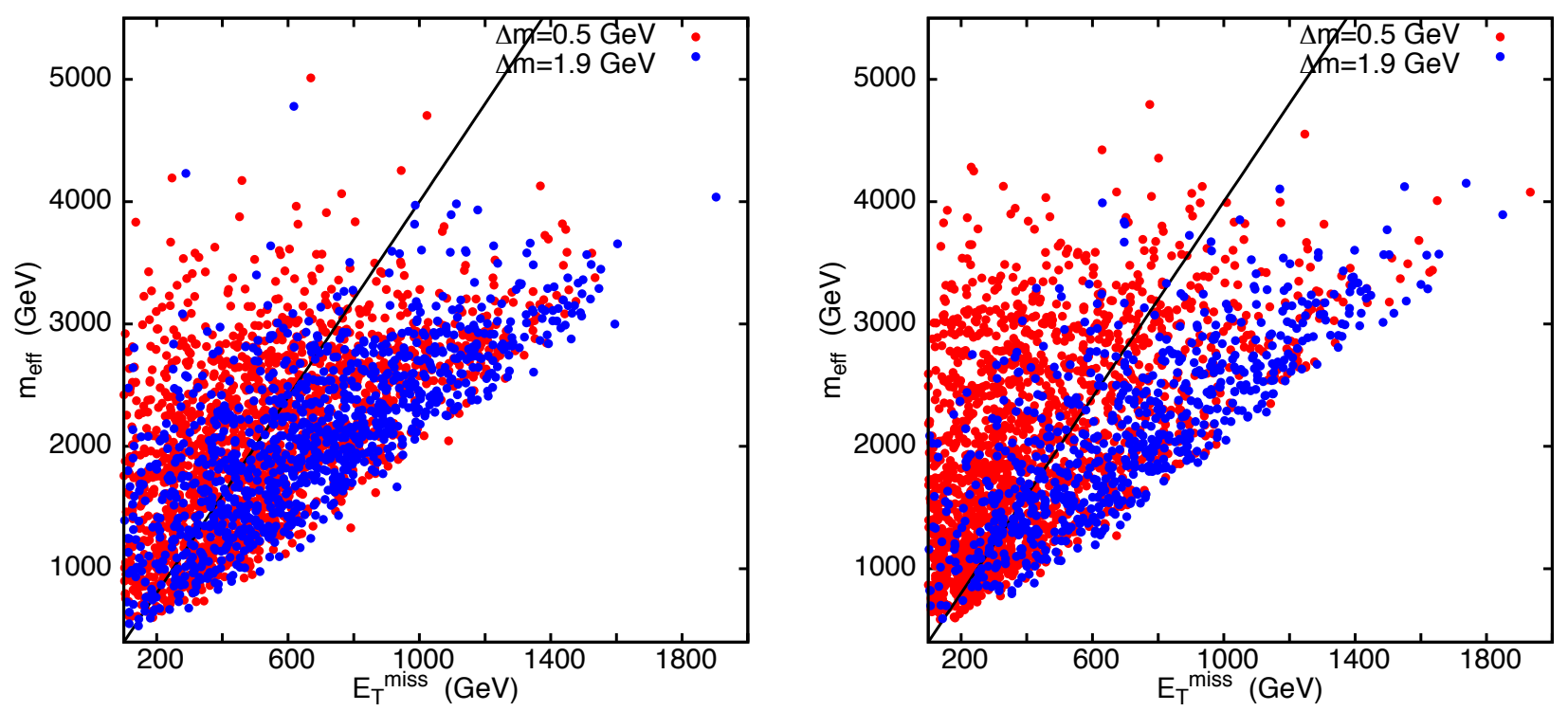

Figure 4: Scatter plot of $E_{T}^{m i s s}$ and $m_{\text {eff }}$ in the four-jet channel for CMSSM scenarios with metastable staus $(\Delta m=0.5 \mathrm{GeV}$, red points) and with rapid $\tilde{\tau} \rightarrow \tau+\chi$ decays $(\Delta m=1.9$ GeV, blue points). The left plot is for $\tan \beta=10, A_{0}=0$ and the right plot is for $\tan \beta=40$, $A_{0}=2.5 m_{0}$, both with $m_{1 / 2}=800 \mathrm{GeV}$. The solid diagonal lines correspond to the ATLAS cut $E_{T}^{\text {miss }}>0.25 m_{\text {eff }}[16$.

\section{The Impacts of LHC Run 1 Searches on the Coan- nihilation Strip Region}

\subsection{Searches for $E_{T}^{\text {miss }}$ Events}

Both the ATLAS and CMS collaborations have performed dedicated sets of experimental searches for generic new physics signatures with an abundance of missing transverse energy, $E_{T}^{\text {miss }}$, as motivated in particular by supersymmetric models in which the stable lightest supersymmetric particle, commonly chosen to be the neutralino, is a massive dark matter particle. The signatures studied generally include jets, which could originate, e.g., from the pair production and subsequent cascade decays of squarks and gluinos. These searches have been carried out for a range of different final states, some including reconstructed leptons as well as jets tagged as originating from $b$-quarks, for a number of different ranges of the missing transverse energy and the total transverse energy. None of these searches found any significant evidence for new physics exhibiting these signatures in the LHC Run 1 data.

The ATLAS collaboration has provided an interpretation of their data in the context of 
the CMSSM based on the 2012 dataset of 20/fb at a centre of mass energy of $8 \mathrm{TeV}$ [16]. The interpretation is presented in the $\left(m_{0}, m_{1 / 2}\right)$ plane for a fixed value of $\tan \beta=30$ and $A_{0}=2 m_{0}$ (in our convention for the sign of $A_{0}$ ). Several different searches have been discussed in [16], but for the purposes of our study we concentrate on the 0-lepton search with 2-6 jets, as this provides the most stringent limit in the region of the stau coannihilation strip, and is also relatively insensitive to the values of $\tan \beta$ and $A_{0}$, as shown in a previous study 28]. As is shown by ATLAS, the CMSSM interpretation of this search provides a limit $m_{1 / 2}>780 \mathrm{GeV}$ at the $95 \% \mathrm{CL}$ near the stau coannihilation strip where $\Delta m>m_{\tau}{ }^{2}$,

We reproduce the ATLAS analysis in [16] using PYTHIA 8 with realistic smearing functions [17] to take into account detector effects, paying particular attention to the signal efficiencies of simulated points in the CMSSM parameter space close to the stau-coannihilation strip. We have considered the various signal regions defined for the ATLAS search, and studied how the different signal region efficiencies change as functions of the stau-neutralino mass splitting.

In the stau-coannihilation region, we find the strongest exclusions in the three-jet channel $(3 j)$ and four-jet channels $(4 j t)$, in agreement with the ATLAS analysis [16]. Our simulation results in a limit on $m_{1 / 2}>(780,830) \mathrm{GeV}$ in the signal regions $3 j$ and $4 j t$ (as defined in [16]) for the same choice of parameters and agrees well with the upper limits of $m_{1 / 2}>$ $(780,840) \mathrm{GeV}$ reported in the conference note [18] together with the analysis referred above. Combining this study with knowledge of the cross-section, we extrapolate the ATLAS limit into the region where $\Delta m<m_{\tau}$.

This search requires tight cuts on missing transverse energy $\left(E_{T}^{\text {miss }}\right)$ and the effective mass $\left(m_{e f f}\right)$ defined as the sum of the $p_{T}$ of all jets plus the $E_{T}^{m i s s}$. To investigate the variation in sensitivity of this search channel, we compare the distributions in these two variables for $\Delta m$ above and below $m_{\tau}$. As can be seen in the scatter plot in Fig. 4 , there are events satisfying the ATLAS $4 j t$ cuts even when $\Delta m=0.5 \mathrm{GeV}$ and the stau is nearly stable (red points), albeit with a smaller efficiency than for $\Delta m=1.9 \mathrm{GeV}$ (blue points). A similar effect is observed in the $3 j$ signal region on which ATLAS exclusions in this region are based. We quantify in Section 4 the sensitivity of this search for small $\Delta m$, in conjunction with the other searches discussed in Sections 3.2 and 3.3 .

\footnotetext{
${ }^{2}$ We note that the analogous CMS $E_{T}^{\text {miss }}$ analysis could provide a similar sensitivity to the CMSSM parameters, but a CMSSM interpretation is not provided.
} 


\subsection{Searches for Metastable Charged Particles}

For stau lifetimes longer than a few nanoseconds, a significant fraction of staus lives long enough to escape the detector leaving a charged track signature. We can also use the $95 \%$ upper limits on long-lived charged particles at $8 \mathrm{TeV}$ reported by CMS [15] to determine exclusions for small stau-neutralino mass differences $\Delta m$. The upper limits for direct production of stau pairs are between $4.3 \mathrm{fb}$ for a stau mass of $126 \mathrm{GeV}$ and $0.26 \mathrm{fb}$ at $494 \mathrm{GeV}$, after which the limit plateaus. The limits for direct + indirect production (i.e., via cascade decays of other sparticles) are similar. For masses between 126-308 GeV, we interpolate using the discrete set of values given in [15], and beyond this we assume an upper limit of $0.3 \mathrm{fb}$, as suggested by the middle left panel of Fig. 8 of [15].

We calculate the fraction of events with at least one stau that is long-lived enough to escape the CMS detector, but exits within the central region $|\eta|<2.1$ so that a track would be visible. Since the CMS upper limit constrains the total cross section, applying this limit to the restricted range of $|\eta|$ is slightly conservative. We find that a significant fraction of the staus with $\Delta m<1.4 \mathrm{GeV}$ are stable on the scale of the CMS detector. We can rule out all $m_{1 / 2}$ values up to about $700 \mathrm{GeV}$, corresponding to $m_{\chi}=291 \mathrm{GeV}$, for $\Delta m<1.4 \mathrm{GeV}$ by the direct production constraint. The excusion tapers off for $\Delta m>1.4 \mathrm{GeV}$, as can be seen in Fig. 1. The maximum $m_{1 / 2}$ ruled out at low $\Delta m$ is between $800-850 \mathrm{GeV}$, which corresponds to a stau mass of $336-345 \mathrm{GeV}$. This agrees with the lower limit of $339 \mathrm{GeV}$ for the mass of a stable stau established by the CMS direct search constraint.

When looking at both direct and indirect production, the search is sensitive to $\Delta m<$ $1.6 \mathrm{GeV}$ for $A_{0}=0$ and $\Delta m<1.7 \mathrm{GeV}$ for $A_{0}=2.5 m_{0}$. The maximum $m_{1 / 2}$ exclusion is between $930 \mathrm{GeV}$ (for $\tan \beta=10, A_{0}=0$ ) and $1100 \mathrm{GeV}$ for both values of $\tan \beta$ with $A_{0}=2.5 m_{0}$. This corresponds to stau masses between $385-447 \mathrm{GeV}$, and is conservative compared to the corresponding upper limit reported by CMS of $500 \mathrm{GeV}$.

\subsection{Searches for Disappearing Tracks}

The disappearing track search by the ATLAS collaboration looks for well-defined tracks that do not proceed beyond the transition radiation tracker (TRT) region of the detector. This corresponds to a radial range of 563-1066 mm and a pseudo-rapidity range of $|\eta|<2.0$. We simulate all event selection cuts in [19], namely (1) $E_{T}^{\text {miss }}>70 \mathrm{GeV}$, (2) $p_{T}^{\text {jet }}>80 \mathrm{GeV}$ and (3) $\Delta \phi_{\min }^{\text {jet }-E_{T}^{\text {miss }}}>$ 1.0. Several further cuts are applied to the stau track - (1) We require the track to be isolated by demanding that the sum of the $p_{T}$ of all charged tracks within a cone of 0.4 around the stau track is less than 0.04 times the $p_{T}$ of the track; (2) 
The candidate track has $p_{T}>15 \mathrm{GeV}$ and is the highest $p_{T}$ track in the event; (3) The track has pseudo-rapidity in the range $0.1<|\eta|<1.9$. For the disappearing track criterion, we demand that the stau decays within the radial and pseudo-rapidity range of the TRT detector.

After applying all the cuts, we validate our simulation by reproducing to within $10 \%$ the efficiency for the benchmark Anomaly Mediated Supersymmetry-Breaking (AMSB) point reported in the analysis. As seen from Fig. 3, a value of $\Delta m$ between 1.4 and $1.77 \mathrm{GeV}$ results in stau lifetimes between 1 and $100 \mathrm{~ns}$, which is the ideal range for disappearingtrack signatures at the LHC.

The $95 \%$ cross-section upper limits reported by ATLAS for events with $p_{T}^{\text {track }}>75,100,150$ and $200 \mathrm{GeV}$ are $1.76 \mathrm{fb}, 1.02 \mathrm{fb}, 0.62 \mathrm{fb}$ and $0.44 \mathrm{fb}$ respectively, which we apply to the cross section for events passing all the cuts (1), (2) and (3) enumerated above. We find that restricting to direct-stau production does not yield any exclusions for $m_{1 / 2}>300 \mathrm{GeV}$. However, including both direct and indirect production, a small region below $m_{1 / 2}=400$ $\mathrm{GeV}$ is ruled out for $\Delta m>1.2 \mathrm{GeV}$ for $A_{0}=0$ and for $\Delta m>1.6 \mathrm{GeV}$ for $A_{0}=2.5 m_{0}$. The jets $+E_{T}^{m i s s}$ search described above provides much stronger constraints for such values of $\Delta m$, excluding $m_{1 / 2}<760 \mathrm{GeV}$ in this region.

\section{Combination of LHC Constraints}

We now discuss the interplay of the various LHC constraints displayed in Fig. 1. The solid maroon lines mark the boundary of the region still allowed following the ATLAS $E_{T}^{m i s s}$ searches at $8 \mathrm{TeV}$. As discussed previously, the most relevant search is that for jets $+E_{T}^{\text {miss }}$, which provides a limit at $m_{1 / 2}=780 \mathrm{GeV}$ in the region where $\Delta m>m_{\tau}$. This constraint is weakened when $\Delta m<m_{\tau}$, as discussed earlier.

The fraction of staus in the final state depends on the cascade decay branching ratios of the heavier sparticles, which depend in turn on the CMSSM parameters, as seen in Fig. 5. For $\tan \beta=40$ and $A_{0}=2.5 m_{0}$, for example, both the second lightest neutralino, $\chi_{2}^{0}$, and the lighter chargino, $\chi_{1}^{ \pm}$, decay almost entirely into final states containing a stau, whereas for $\tan \beta=10$ and $A_{0}=0$ they decay into staus in only about $20 \%$ and $64 \%$ of the cases, respectively. However, even when the stau lifetime is long enough that most staus escape the detector, sensitivity to $E_{T}^{\text {miss }}$ is lost only if the decay chains of both the produced sparticles result in staus. The dependence on $\Delta m$ of the fraction of staus stable enough to exit the CMS detector is shown in the left panel of Fig. 5. As expected, we find that for small $\Delta m$, most staus are stable. The dashed lines (which correspond to the stau fraction from both 
direct and indirect production) asymptote to the total stau fraction in the final states as $\Delta m \rightarrow 0$. As $\Delta m$ increases, the fraction of stable staus decreases until it becomes zero at the tau mass threshold. We find that when all production processes and decay chains are taken into account, the loss in efficiency for the $E_{T}^{\text {miss }}$-based search does not differ significantly for different values of $\tan \beta$ and $A_{0}$, and the limit $m_{1 / 2}>780 \mathrm{GeV}$ given in [16] reduces to about $750 \mathrm{GeV}$.
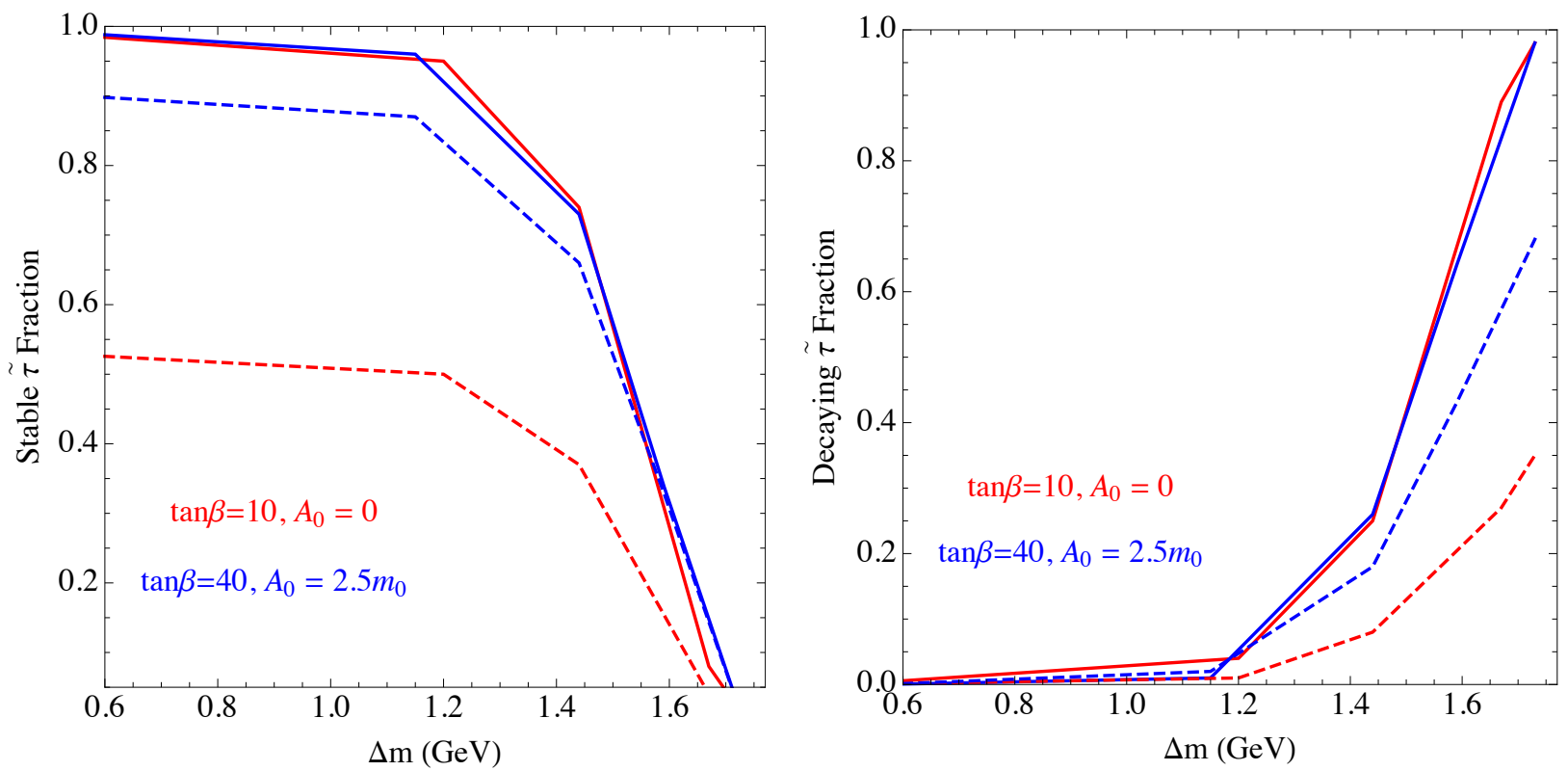

Figure 5: The dependence of the fraction of 'stable' staus, i.e., those exiting CMS without decaying (left), and the fraction of staus decaying within the ATLAS detector (right) on the $\tilde{\tau}-\chi$ mass difference. The solid lines correspond to direct stau-pair production only whereas the dashed are for both direct and indirect stau production (i.e., all SUSY processes). The value of $m_{1 / 2}$ is fixed to $800 \mathrm{GeV}$.

We find that the most important constraint in the band where $\Delta m<m_{\tau}$ is that due to the search for metastable charged particles, shown by the shaded blue regions in Fig. 1. The darker shading is the constraint from direct stau pair production, and the lighter shading is that obtained by including indirect stau production via the cascade decays of heavier sparticles. The direct constraint yields a lower limit $m_{1 / 2} \gtrsim 700 \mathrm{GeV}$ for $\Delta m \lesssim 1.4 \mathrm{GeV}$, which is only weakly sensitive to $\tan \beta$ but becomes stronger for $\Delta m \lesssim 1 \mathrm{GeV}$ when $A_{0}=$ $2.5 m_{0}$. The indirect constraint yields a lower limit that is somewhat more sensitive to both $\tan \beta$ and $A_{0}$, yielding a lower limit $m_{1 / 2} \gtrsim 800$ to $1100 \mathrm{GeV}$. 
As can be seen from the right panel of Fig. 5, the search for disappearing tracks becomes relevant for $m_{\tau}>\Delta m \gtrsim 1.2 \mathrm{GeV}$. However, as already noted, it is weaker than the other constraints, yielding $m_{1 / 2} \gtrsim 400 \mathrm{GeV}$. This is mainly because, even with a significant fraction of staus decaying before exiting the detector, the signal efficiency for this search is of the order of $0.1-0.01 \%$ after implementing the cuts enumerated in section 3.3 .

In the case of $\tan \beta=10$, for both $A_{0}=0$ and $2.5 m_{0}$ we see that the portions of the coannihilation strips with $\Delta m \gtrsim 3 \mathrm{GeV}$ are excluded by the ATLAS jets $+E_{T}^{\text {miss }}$ search, whereas the portions with $3 \mathrm{GeV} \gtrsim \Delta m>m_{\tau}$ are allowed by this search. For $\tan \beta=40$ and $A_{0}=0\left(2.5 m_{0}\right)$ the portion of the strip where $6 \mathrm{GeV}(9 \mathrm{GeV}) \gtrsim \Delta m>m_{\tau}$ is allowed by this search. When $\Delta m<m_{\tau}$, we see that none of the strips for $\tan \beta=10, A_{0}=0$ or $\tan \beta=40$ and $A_{0}=0$ or $2.5 m_{0}$ can be excluded, whereas for $\tan \beta=10$ and $A_{0}=2.5 m_{0}$ the portion of the strip with $\Delta m \lesssim 1.7 \mathrm{GeV}$ is excluded by the search for massive charged particles.

In the ranges of $m_{1 / 2}$ exhibited in Fig. 1, FeynHiggs 2.10.0 generally yields values of $m_{h}$ below the value measured at the LHC. Taking into account the uncertainties in the theoretical calculation of $m_{h}$, points yielding a nominal value $\sim 122.5 \mathrm{GeV}$ should probably not be regarded as excluded. Even taking this uncertainty into account, only the case $\tan \beta=40, A_{0}=2.5 m_{0}$ is consistent with the LHC measurement of $m_{h}{ }^{3}$.

\section{The Potential Reaches of Future LHC Searches}

In order to extrapolate the potential reach of each of the three distinct categories of searches used in the analysis of this paper, we make a simple but, we believe, reasonable assumption, namely that the expected cross-section sensitivities of the respective searches will remain the same when going from a centre-of-mass energy of $8 \mathrm{TeV}$ to 13 or $14 \mathrm{TeV}$. Based on this assumption, we use PYтнiA 8 [20,21] to recalculate the cross-sections in this higher centre-of-mass regime, and extrapolate the mass limit by requiring that, at the new mass limit, the cross-section multiplied by the respective integrated luminosity is the same as for the Run 1 LHC data. The results of applying this hypothesis are shown in Fig. 6.

Fig. 6 compares the prospective limits with the tip of the stau coannihilation strip for $\tan \beta=40$ and $A_{0}=2.5 m_{0}$, which was shown in Fig. 1 to be the most difficult to exclude. We do not display the projected sensitivity of the 'disappearing track' search, which we do

\footnotetext{
${ }^{3}$ We have checked that the value of $m_{h}$ calculated using SoftSUSY 3.3.7 is $\sim 1.5 \mathrm{GeV}$ smaller than the value given by FeynHiggs 2.10.0 for the parameter ranges presented in Fig. 1. This difference is within the latter code's uncertainties.
} 


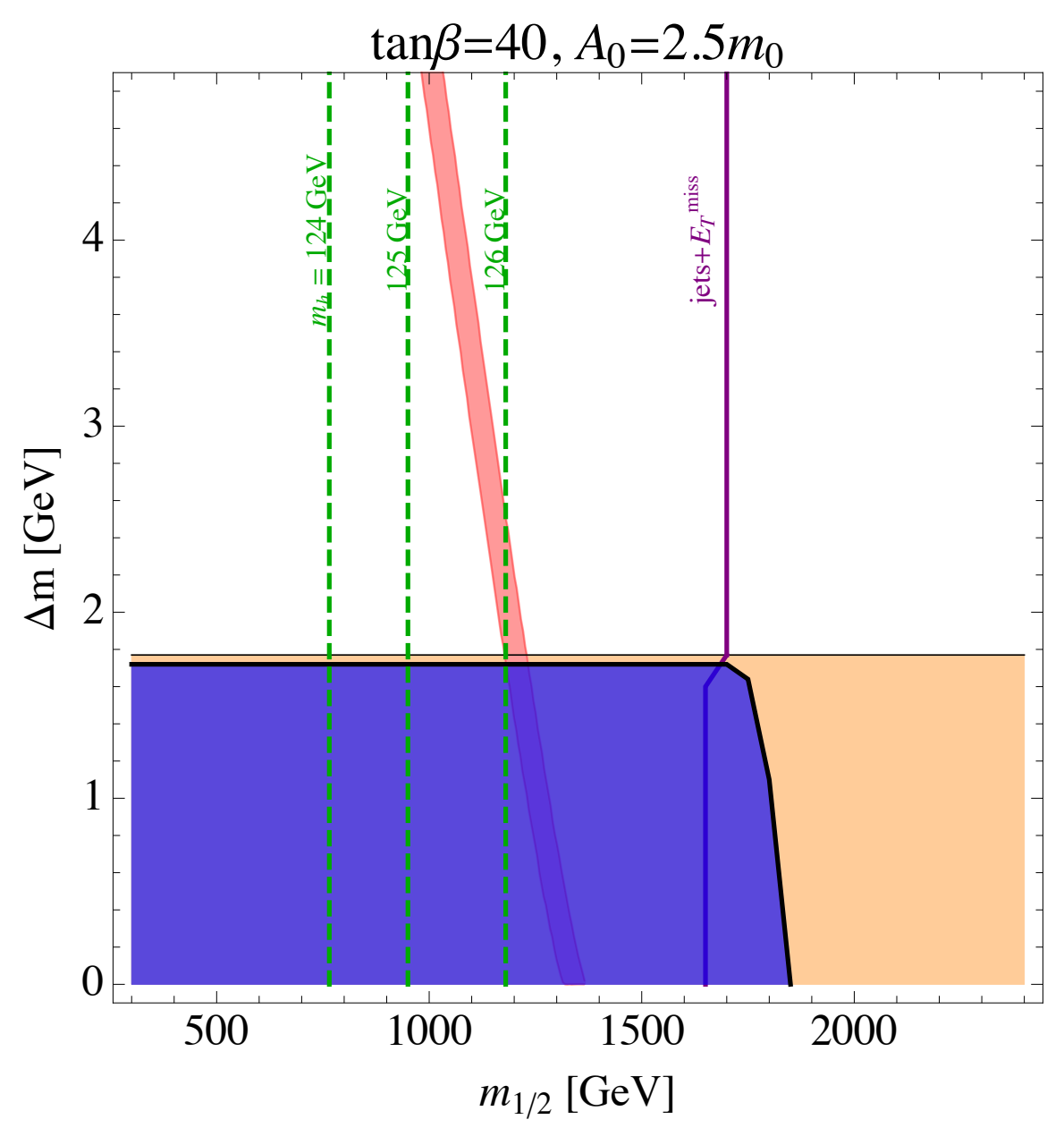

Figure 6: Projected limits from the 14 TeV LHC Run 2 with 300/fb integrated luminosity. The sensitivity of the jets $+E_{T}^{\text {miss }}$ search is sufficient to explore the rest of the coannihilation strip, and the search for metastable charged tracks from direct stau-pair production is also strong enough to explore independently the portion of the coannihilation strip where $\Delta m \lesssim$ $1.6 \mathrm{GeV}$.

not expect to be competitive because of its low efficiency, as discussed earlier. We see that the projected sensitivity of the jets $+E_{T}^{\text {miss }}$ search is $\sim 1700 \mathrm{GeV}$ for $\Delta m>m_{\tau}$, decreasing to $\sim 1650 \mathrm{GeV}$ for $\Delta m<1.6 \mathrm{GeV}$, which is sufficient to explore all the coannihilation strip. The most sensitive search channel when $\Delta m \lesssim 1.6 \mathrm{GeV}$ is expected to be that for massive charged particles, which would also be strong enough to explore independently this portion of the coannihilation strip: its sensitivity should reach $\sim 1850 \mathrm{GeV}$ for very small $\Delta m$. The combination of these searches would clearly explore thoroughly the CMSSM coannihilation strip in this and, a fortiori, the other cases we study. Indeed, we estimate the tip of the strip at $m_{1 / 2} \sim 1400 \mathrm{GeV}$ can be explored with $75 / \mathrm{fb}$ data at $14 \mathrm{TeV}$. Points within the 
stau coannihilation strip could be detected in two ways, via their $E_{T}^{\text {miss }}$ and massive stable particle signatures. Conversely, the absence of signals in both these channels would exclude robustly the stau coannihilation strip.

\section{Conclusions}

We have analyzed in this paper the impacts on the CMSSM parameter space in the neighbourhood of the tip of the stau coannihilation strip of various LHC searches, including the ATLAS jets $+E_{T}^{\text {miss }}$ search, the CMS search for massive charged particles, and the ATLAS search for disappearing tracks, which are sensitive in different regions of $\Delta m$ and $m_{1 / 2}$. We have found that the jets $+E_{T}^{m i s s}$ search has important sensitivity when $\Delta m<m_{\tau}$, though the strongest constraint for small $\Delta m$ is generally that provided by the search for massive charged particles. The search for disappearing tracks has impact only for $\Delta m \gtrsim 1.6 \mathrm{GeV}$, where it is considerably less sensitive than the jets $+E_{T}^{\text {miss }}$ search.

We have studied four CMSSM cases, with the following conclusions. For $\tan \beta=10$ and $A_{0}=0$, the portion of the coannihilation strip with $\Delta m \gtrsim 3 \mathrm{GeV}$ is excluded by the jets $+E_{T}^{\text {miss }}$ search, but the portion with $\Delta m \lesssim 3 \mathrm{GeV}$ cannot yet be excluded. For $\tan \beta=10$ and $A_{0}=2.5 m_{0}$, the portion of the coannihilation strip with $\Delta m \gtrsim 3 \mathrm{GeV}$ is again excluded by the jets $+E_{T}^{\text {miss }}$ search, and the portion with $\Delta m \lesssim 1.7 \mathrm{GeV}$ is excluded by the search for massive charged particles, but there is no exclusion for the portion with $1.7 \mathrm{GeV} \lesssim \Delta m \lesssim 3 \mathrm{GeV}$. For $\tan \beta=40$ and $A_{0}=0$, only the portion of the coannihilation strip with $\Delta m \gtrsim 6 \mathrm{GeV}$ is excluded, again by the jets $+E_{T}^{\text {miss }}$ search, and there is no exclusion for $\Delta m<m_{\tau}$. Finally, for $\tan \beta=40$ and $A_{0}=2.5 m_{0}$, only the portion of the coannihilation strip with $\Delta m \gtrsim 9 \mathrm{GeV}$ is excluded, again by the jets $+E_{T}^{\text {miss }}$ search.

We have also projected the likely sensitivities of the LHC searches in Run 2 of the LHC at energies approaching $14 \mathrm{TeV}$ and with up to 300/fb of integrated luminosity. We find that a combination of the jets $+E_{T}^{\text {miss }}$ and massive charged particle searches should be able to explore robustly the entire CMSSM coannihilation strip for all the cases we have studied. The end of the CMSSM coannihilation strip is indeed nigh, one way or another.

\section{Acknowledgements}

This work was supported partly by the London Centre for Terauniverse Studies (LCTS), using funding from the European Research Council via the Advanced Investigator Grant 267352. The work of J.E. was also supported in part by the UK STFC via the research 
grant ST/J002798/1. N.D. would like to thank the Alexander von Humboldt Foundation for support.

\section{References}

[1] ATLAS Collaboration, https://twiki.cern.ch/twiki/bin/view/AtlasPublic/SupersymmetryPublicResults.

[2] CMS Collaboration, https://twiki.cern.ch/twiki/bin/view/CMSPublic/PhysicsResultsSUS.

[3] H. Goldberg, Phys. Rev. Lett. 50 (1983) 1419; J. Ellis, J. Hagelin, D. Nanopoulos, K. Olive and M. Srednicki, Nucl. Phys. B 238 (1984) 453.

[4] E. Komatsu et al. [WMAP Collaboration], Astrophys. J. Suppl. 192 (2011) 18 arXiv:1001.4538 [astro-ph.CO]].

[5] P. A. R. Ade et al. [Planck Collaboration], arXiv:1303.5076 [astro-ph.CO].

[6] M. Drees and M. M. Nojiri, Phys. Rev. D 47 (1993) 376 arXiv:hep-ph/9207234]; H. Baer and M. Brhlik, Phys. Rev. D 53 (1996) 597 arXiv:hep-ph/9508321]; Phys. Rev. D 57 (1998) 567 |arXiv:hep-ph/9706509]; H. Baer, M. Brhlik, M. A. Diaz, J. Ferrandis, P. Mercadante, P. Quintana and X. Tata, Phys. Rev. D 63 (2000) 015007 arXiv:hepph/0005027.

[7] G. L. Kane, C. F. Kolda, L. Roszkowski and J. D. Wells, Phys. Rev. D 49 (1994) 6173 arXiv:hep-ph/9312272]; J. R. Ellis, T. Falk, K. A. Olive and M. Schmitt, Phys. Lett. B 388 (1996) 97 arXiv:hep-ph/9607292]; Phys. Lett. B 413 (1997) 355 arXiv:hepph/9705444]; J. R. Ellis, T. Falk, G. Ganis, K. A. Olive and M. Schmitt, Phys. Rev. D 58 (1998) 095002 arXiv:hep-ph/9801445]; V. D. Barger and C. Kao, Phys. Rev. D 57 (1998) 3131 arXiv:hep-ph/9704403 ; J. R. Ellis, T. Falk, G. Ganis and K. A. Olive, Phys. Rev. D 62 (2000) 075010 arXiv:hep-ph/0004169].

[8] J. Ellis, T. Falk, and K.A. Olive, Phys. Lett. B444 (1998) 367 arXiv:hep-ph/9810360]; J. Ellis, T. Falk, K.A. Olive, and M. Srednicki, Astr. Part. Phys. 13 (2000) 181 [Erratum-ibid. 15 (2001) 413] arXiv:hep-ph/9905481]. 
[9] C. Boehm, A. Djouadi and M. Drees, Phys. Rev. D 62 (2000) 035012 hep-ph/9911496;

J. R. Ellis, K. A. Olive and Y. Santoso, Astropart. Phys. 18 (2003) 395 hepph/0112113]; J. Ellis, K. A. Olive and J. Zheng, arXiv:1404.5571 [hep-ph].

[10] A. B. Lahanas, D. V. Nanopoulos and V. C. Spanos, Mod. Phys. Lett. A 16 (2001) 1229 arXiv:hep-ph/0009065; A. B. Lahanas and V. C. Spanos, Eur. Phys. J. C 23 (2002) 185 arXiv:hep-ph/0106345; J. R. Ellis, T. Falk, G. Ganis, K. A. Olive and M. Srednicki, Phys. Lett. B 510 (2001) 236 arXiv:hep-ph/0102098].

[11] J. L. Feng, K. T. Matchev and T. Moroi, Phys. Rev. Lett. 84, 2322 (2000) arXiv:hepph/9908309; Phys. Rev. D 61, 075005 (2000) arXiv:hep-ph/9909334; J. L. Feng, K. T. Matchev and F. Wilczek, Phys. Lett. B 482, 388 (2000) arXiv:hep-ph/0004043.

[12] M. Citron, J. Ellis, F. Luo, J. Marrouche, K. A. Olive and K. J. de Vries, Phys. Rev. D 87 (2013) 036012 [arXiv:1212.2886 [hep-ph]].

[13] T. Cohen and J. G. Wacker, JHEP 1309 (2013) 061 arXiv:1305.2914 [hep-ph]].

[14] Y. Konishi, S. Ohta, J. Sato, T. Shimomura, K. Sugai and M. Yamanaka, arXiv:1309.2067 [hep-ph].

[15] S. Chatrchyan et al. [CMS Collaboration], JHEP 1307 (2013) 122 arXiv:1305.0491 [hep-ex]].

[16] G. Aad et al. [ATLAS Collaboration], arXiv:1405.7875 [hep-ex].

[17] S. Chatrchyan et al. [CMS Collaboration] CMS-DP-2013-003; CMS-DP-2013-033; JINST 8, P09009 (2013); JINST 7 P10002 (2012).

[18] The ATLAS collaboration, ATLAS-CONF-2013-047.

[19] G. Aad et al. [ATLAS Collaboration], Phys. Rev. D 88 (2013) 112006 arXiv:1310.3675 [hep-ex]].

[20] T. Sjostrand, S. Mrenna and P. Z. Skands, Comput. Phys. Commun. 178 (2008) 852 arXiv:0710.3820 [hep-ph]].

[21] N. Desai and P. Z. Skands, Eur. Phys. J. C 72 (2012) 2238 [arXiv:1109.5852 [hep-ph]].

[22] G. Aad et al. [ATLAS Collaboration], Phys. Lett. B 716, 1 (2012) arXiv:1207.7214 [hep-ex]]. 
[23] S. Chatrchyan et al. [CMS Collaboration], Phys. Lett. B 716, 30 (2012) arXiv:1207.7235 [hep-ex]].

[24] T. Hahn, S. Heinemeyer, W. Hollik, H. Rzehak and G. Weiglein, arXiv:1312.4937 [hep$\mathrm{ph}$.

[25] B. C. Allanach, Comput. Phys. Commun. 143 (2002) 305 [hep-ph/0104145].

[26] Information about this code is available from K. A. Olive: it contains important contributions from T. Falk, A. Ferstl, G. Ganis, F. Luo, A. Mustafayev, J. McDonald, K. A. Olive, P. Sandick, Y. Santoso and M. Srednicki.

[27] G. Belanger, F. Boudjema, A. Pukhov and A. Semenov, Comput. Phys. Commun. 185 (2014) 960 [arXiv:1305.0237 [hep-ph]]; Comput. Phys. Commun. 174 (2006) 577 hepph/0405253; Comput. Phys. Commun. 149 (2002) 103 hep-ph/0112278.

[28] O. Buchmueller, R. Cavanaugh, M. Citron, A. De Roeck, M. J. Dolan, J. R. Ellis, H. Flacher and S. Heinemeyer et al., Eur. Phys. J. C 72 (2012) 2243 arXiv:1207.7315, see also O. Buchmueller, R. Cavanaugh, A. De Roeck, M. J. Dolan, J. R. Ellis, H. Flacher, S. Heinemeyer and G. Isidori et al., Eur. Phys. J. C 74 (2014) 2922 arXiv:1312.5250 [hep-ph]].

[29] T. Jittoh, J. Sato, T. Shimomura and M. Yamanaka, Phys. Rev. D 73, 055009 (2006) hep-ph/0512197. 